\title{
Artificial Intelligence: A New Way to Improve Indian Agriculture
}

\author{
Diksha Manaware* \\ Department of Horticulture (Vegetable Science), JNKVV, Jabalpur (M.P.), India \\ *Corresponding author
}

\section{A B S T R A C T}

Agriculture, with its allied sectors, is unquestionably the largest livelihood

Keywords

Sectors,

rural India , crops, weather

Article Info

Accepted:

05 February 2020

Available Online:

10 March 2020 provider in India; more so in the vast rural India. Agriculture plays a vital role in Indian economy. Government has set a target of doubling of farmer's income by the year 2022 as well as Agriculture export policy has set a target to increase agricultural exports to over US\$ 60 billion by 2022 . The digital technology can play a transformational role in modernizing and organizing how rural India performs its agricultural activities. The technologies include Artificial Intelligence, Big Data Analytics, Block Chain Technology, Internet of Things etc. Artificial Intelligence provides accurate and timely information regarding crops, weather and insect etc. to the farmers may improve the crop productivity, reduce the risk and improve the income of the farmers.

\section{Introduction}

Artificial intelligence (AI) is a branch of computer science concerned with building smart machines capable of performing tasks that typically require human intelligence. The "AI" term was coined by john McCarthy, an American computer scientist, back in 1956 at The Dartmouth Conference. The term artificial intelligence composed of word "artificial" (made or produced by human being rather than occurring naturally) and "intelligence" (the ability to acquire and apply knowledge and skills).
Artificial intelligence (AI) makes it possible for machines to learn from past experience, adjust to new inputs and have the ability to execute tasks naturally associated with human intelligence, like speech recognition, decision- making, visual perception and translating languages.

\section{Types of artificial intelligence}

\section{Artificial narrow intelligence (ANI)}

ANI refers to a machine's ability to perform specific task autonomously using human-like capabilities. eg. Google map, chatbot. 


\section{Artificial general intelligence (AGI)}

AGI refers to a machine that can understand or learn any intellectual task that a human being can.

\section{Artificial super intelligence (ASI)}

ASI is smarter than the collective intellect of the smartest humans in every field. Artificial intelligence works at its best by combining large amounts of data sets with fast, repetitive processing and intelligent algorithms. This makes possible for the AI software to learn automatically from patterns in those vast data sets.

The terms Artificial Intelligence, Machine learning and Deep learning all are used interchangeably, however machine learning is a subset of AI, and consist of the more advanced techniques and models that enable computers to figure things out from the data while deep learning is a subset of machine learning that uses multi-layered artificial neural networks to deliver high accuracy in tasks such as speech recognition, language translation and object detection etc.

\section{Why artificial intelligence is playing important role in Indian agriculture?}

Agriculture is the most important sector of Indian economy. Indian agriculture sector accounts for 18 per cent of India's gross domestic product (GDP) and ensure food security to roughly 1.3 billion people. India has many areas to choose for business such as dairy, meat, poultry, fisheries and food grain etc.

Agricultural exports constitute 10 percent of the country's exports and are the fourth largest exported principal commodity category in India. India still depends on resource intensive agriculture practices. Major problems such as degradation of land, increased dependence on inorganic fertilizers, reduction in soil fertility, reduction in ground water table and pest resistance etc are clear indication for India's unsustainable agricultural practices.

As climate change becomes more sensible and unpredictable, dependence on unsustainable agriculture practices will only increase the risk of food scarcity. In a similar way, use of water in agriculture continues to be high and sub-optimal. In spite of having just one-third of the gross cropped area under irrigation, agriculture use $89 \%$ of extracted groundwater.

On the other hand absence of functional endto-end agriculture value chains has caused the price realization. Artificial Intelligence technologies are helpful to yield healthier crops, provides information of prevailing weather conditions such as temperature, rain, weed speed, weed direction and solar radiation, control pests, monitor soil and growing conditions, organize data for farmers, help with workload and improve food supply chain. India has $\sim 30$ million farmers who own smart phones, which is expected to grow three times by 2020 and 315 million rural Indians will be using internet by 2020. It is estimated that AI and connected farm services can impact 70 million farmers by 2020 , thereby adding US\$ 9 billion to farmer's income.

AI is an important part of the Precision Agriculture. The goal of the precision agriculture is to define a decision support system (DSS) for whole farm management with the goal of optimizing returns on inputs while preserving resources. This practice has been enabled by the advent of GPS and GNSS. The growing introduction of complex algorithms, robotics, sensors and satellites shows that AI has made its mark in precision agriculture. 


\section{Applications of artificial intelligence (AI) in Indian agriculture}

\section{Crop health monitoring}

Assessment of the health of a crop, as well as early detection of crop infestations, is critical in ensuring good agricultural productivity. Stress associated with, such as climate change, nutrient deficiencies, weed, insect and fungal infestations must be detected early enough to provide an opportunity for the farmers to mitigate. Ai can be used to predict advisories for sowing, pest control, input control can help in ensuring increased income and providing stability for the agricultural community. Using high resolution weather data, remote sensing data, AI technologies and AI platform, it is possible to monitor crops holistically and provide additional insights to to the farmers for their farms as and when required.

\section{Microsoft, ICRISAT develop sowing application for farmers using AI}

A sowing application for farmers combined with a personalized village advisory dashboard for Andhra Pradesh has been developed by Microsoft India in collaboration with International Crops Research Institute for Semi- Arid Tropics (Icrisat). The sowing app advises farmers on the best time to sow crops depending on weather conditions, soil and other indicators. The sowing app is developed to provide powerful cloud-based predictive analytics to empower farmers with crucial information and insights to help reduce crop failure and increase yield, in turn, reducing stress and generating better income.

\section{Soil health monitoring}

Soil is for the farmer what the pulse is for the doctor. It helps them take decisions about when to irrigate, when and what to sow, nutrient application and so on. Image recognition and deep learning models have enabled distributed soil health monitoring without the need of laboratory testing infrastructure. AI solutions integrated with data signals from remote satellites, as well as local image capture in the farm and help farmers to take immediate possible action to restore the soil health.

\section{Soilsens}

A technology called soilsens is a low cost smart soil monitoring system has come as a potential help to farmers facing farming decisions predicament. This technology is developed by Proximal Soilsens Technologies Pvt. Ltd, a startup incubated at Indian Institute of Technology Bombay (IITB), Mumbai with support from the Ministry of Department of Science and Technology (DST) and Ministry of Electronics and Information Technology. The system is embedded with soil moisture sensor, soil temperature sensor, ambient humidity sensor and ambient temperature sensor. Based on this parameters, farmers are advised about optimum irrigation through a mobile app. This data is also available on cloud. The technology can help improve efficiency of water usage in agriculture. It can help with guidance about ways to optimize water usage as per the requirement of the crop and soil. The system can also help to avoid over irrigation, thus protecting crops from disease, prevent leaching of nutrients from the soil, saving water, electricity, predict early onset of diseases and offer advisories.

\section{Plantix app}

Berlin-based agricultural tech startup PEAT has developed a deep learning application called plantix that identifies potential defects and nutrient deficiencies in soil. The analysis is conducted by software algorithms which correlate particular foliage patterns with 
certain soil defects, plant pest and disease. The app uses images to detect plant diseases and other possible defects through images captured by the user's smart phone camera. It is also offers corresponding treatment measures.

\section{Agricultural robotics and drones}

Agriculture robotics also known as an agribot, now becoming popular due to labor shortages and increased need to feed the global population. Agribots automate tasks for farmers, increasing the efficiency of production and reducing the industry's dependency on manual labor. This includes applications such as harvesting; picking, seeding, spraying, pruning, sorting and packing etc.

Drones are equipped with multi-spectral and photo cameras that can monitor crop stress, plant growth and predict yield. It is time and labour saving technology in not having to go out to visual checking on a crop. The more advanced drones can carry and deliver payloads like herbicide, fertilizer and water.

\section{Robot drone tractor}

Robot will decide where to plant, when to harvest and how to choose the best route for crisscrossing the farmland. These robots are to reduce the usage of pesticides, herbicides, fertilizers and water.

\section{Predictive analytics}

With climate change, forecasts are now important for crop yields as farmers cannot end just on traditional knowledge. More accurate forecasts could enable farmers to pick the optimal days for planting or harvesting. AI techniques apply reinforcement learning on past predictions and actual outcomes. To aid in weather forecasting, data is fed into an algorithm that uses deep learning techniques to learn and make predictions based on past data.

\section{Weather forecasting}

Artificial Intelligence in farming along with the satellite data can be used to predict the weather conditions analyze the crop sustainability and evaluate the farms for the presences of pests and diseases. The Artificial Intelligence (AI) in farming is able to provide billions of data points including temperature, precipitation, wind speed and solar radiation.

\section{Supply chain efficiencies}

Using AI, farmers would be able to understand market demand for their produce and also customer's choices and seasonality. This will help the farmers to get better return from their produce. AI-powered supply chains, on the other hand, can help improve their bottom line by reducing the cost incurred in managing distributed logistics and a multitude of middlemen. Through this smart routing, smaller farmers too will be able to organize their route to market more efficiently and gain benefits. They would also be able to get their perishable goods to market quicker without intervention of middlemen thus reducing wastage and losses.

\section{Jivabhumi}

Jivabhumi is an agri- tech platform for connecting farmers directly with Institutional buyers and consumers. Jivabhumi partners with farmers, farmers group, aggregates farm produce and makes it traceable leveraging BLOCKCHAIN enabled platform called FOOTPRINT. Jivabhumi enables consumers (B2C) and institutional buyers (B2B) to buy chemical, pesticide free and traceable farm produce directly from the producers. Jivabhumi is accelerated by India's leading 
start-up accelerators such as YES Scale Accelerator, a-idea NAARM, NSRCELIIMB, KIIT-TBI and a grant recipient from Karnataka Startup Cell. FOODPRINT is a produce aggregation and produce traceability solution which aggregates the farm produce and implements produce traceability using technology such as BLOCKCHAIN to capture the information of the produce at various levels in the supply chain. Its aim is to digitalizing agriculture to solve supply chain ineffiencies using technology.

\section{Gobasco}

Gobasco is an artificial intelligence- based platform that offers procurement optimization and yield prediction solution for the agriculture sector. The aim is to use artificial intelligence and big data to optimize the agrisupply-chain across India. This approach provides farmers and agricultural SMEs (Small and Medium enterprises) with a datarich technology platform and network to grow their profits, thereby creating new opportunities in rural commerce.

\section{National strategy for artificial intelligence}

National Institute for Transforming India, Government of India has partnered with several leading $\mathrm{Ai}$ technology players to implement AI projects in agriculture. The agriculture sector in India, which forms the bedrock of India's economy, needs multilayered technology infusion and coordination amongst several stakeholders; hence require government to play a leading role in developing the implementation roadmap for AI in agriculture. The government of India has recently prioritized Doubling Farmer's Income as National Agenda; putting considerable focus on supply chain perspectives in agriculture and market development in addition to productivity boost.
Artificial intelligence powered projects in Indian agriculture sector

\section{e- National agriculture market (eNAM)}

eNAM is an online trading platform for agricultural commodities in India. The market facilitates farmers, traders and buyers with online trading in commodities. The market is helping in better price discovery and provides facilities for smooth marketing of their produce. Over 90 commodities including staple food grains, vegetables and fruits are currently listed in its list of commodities available for trade. The objectives of e-NAM are to provide transparent sale transactions and price discovery initially in regulated markets. The promotion of e- trading is by the state agricultural marketing board/ Agricultural produce market committee (APMC). It provides liberal licensing of traders/ buyers and commission agents by state authorities without any pre-condition of physical presence and one license for a trader valid across all markets in the state.

\section{AI for precision farming}

The government's policy think-tank NITI Ayog partnered with IBM ( to develop a crop yield prediction model using artificial intelligence (AI) to provide real time advisory to farmers in 10 aspirational districts across the states of Assam, Bihar, Madhya Pradesh, Maharashtra, Rajasthan and Uttar Pradesh. The partnership aims to work together towards use of technology to provide insights to farmers to improve crop productivity, soil yield, control agriculture inputs and early warning on pest/disease outbreak will use data from remote sensing (ISRO), soil health cards, IMD's weather prediction and soil moisture/ temperature, crop phenology etc. to give accurate prescriptions to farmers with the overall goal of improving farmer's income. 
Pradhan mantri fasal bima yojana (PMFBY)

PMFBY will be providing support to farmers who are suffering from crop loss or damage arising out of unforeseen events, along with stabilizing the income of farmers to ensure their continuance in farming. In order to speed up claim settlement of farmers under the existing crop insurance scheme, the agriculture ministry has decided to use specialized agencies to carry out pilot studies to estimate crop yield at village level using innovative technologies like AI, remote sensing imageries and modeling tools.

\section{PM- KISAN}

By leveraging the benefits of AI, Pradhan Mantri Kisan Samman Nidhi is an initiative by the government of India in which all small and marginal farmers will get up to Rs 6,000 (US\$84) per year as minimum income support. The government is aimed to leverage the huge amount of collected data by several agri-schemes and use the same to better target the farmers who requires the benefits of PMKISAN. The data will be used in creating a proper framework for farmers, along with the right policy. It will also help in converging some government projects to achieve the targeted development of farmers and the overall sector.

\section{AGRI-UDAAN}

AGRI-UDAAN is a food and agribusiness accelerator 3.0 organized by a- IDEA, Technology Business Incubator of NAARM, supported by Dept. of Science \& Technology, Government of India. The program focuses on catalyzing scale-up stage food and agribusiness startups through rigorous mentoring, industry networking and investor pitching. This initiative is a 6- month program launched in Hyderabad.

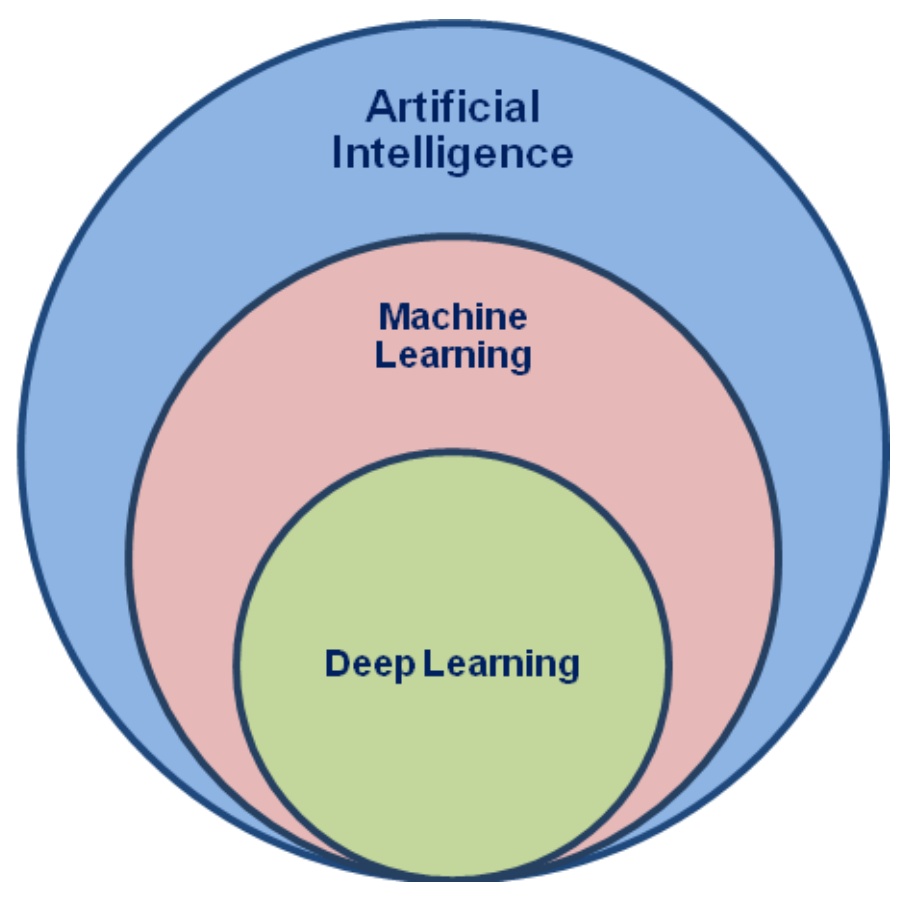

Figrue.1 


\section{How the Sowing App helps farmers increase yields}

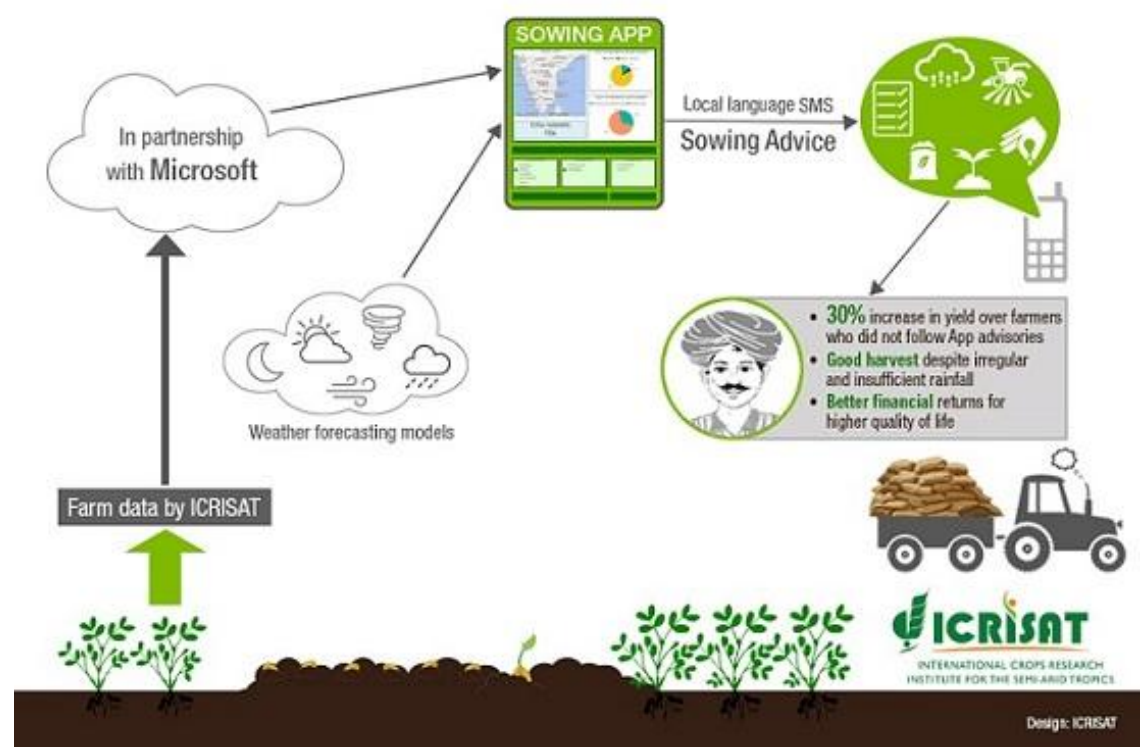

Figure.2 Source: icrisat.org

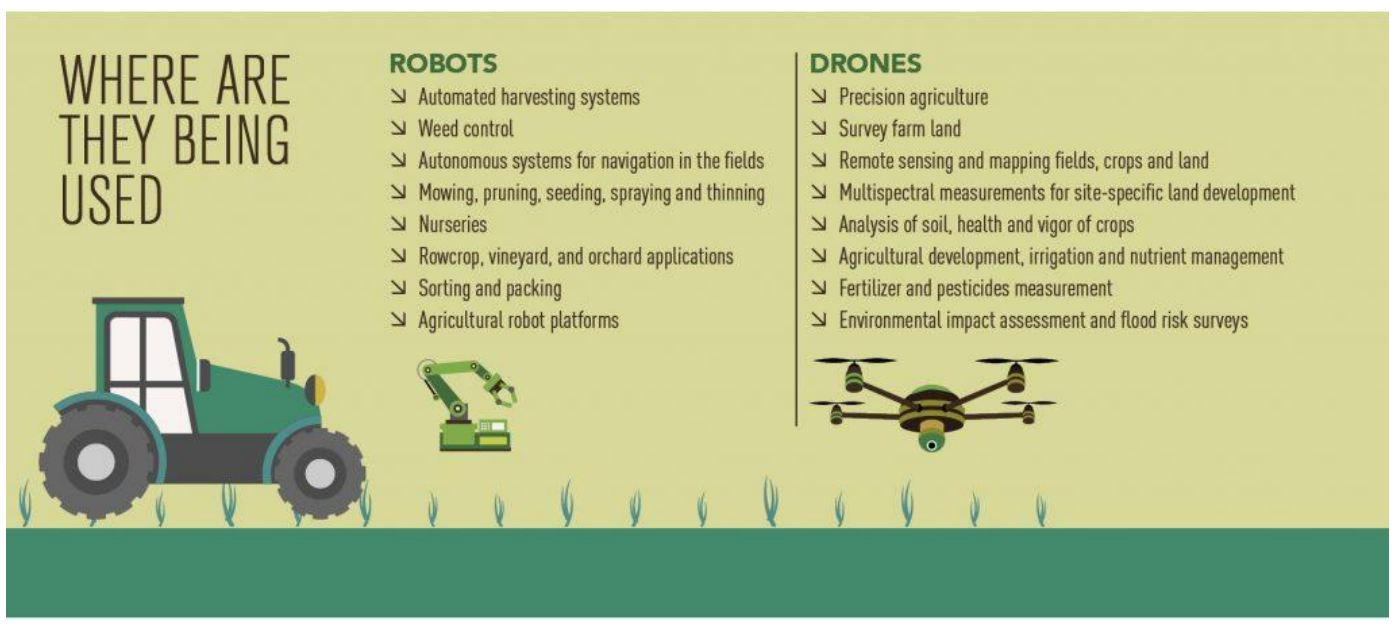

Figure.3 Source: Drones and Robots: Revolutionizing Farms of the Future, Geospatial world

\section{Government of karnataka inks MoU with microsoft}

Government of Karnataka has signed the MoU with the Microsoft Corporation India Private Limited. The collaboration intends to empower smallholder farmers with AI- based solutions that will help them increase income using ground- breaking, cloud-based technologies, machine learning and advanced analytics. Microsoft with guidance from
Karnataka Agricultural Price Commission (KAPC) is aiming to use digital tools to develop a multivariate agricultural commodity price forecasting model considering the following parameters such as sowing area, production time, yielding time, weather datasets etc.

\section{Maha agri tech project}

The first phase of the project uses satellite 
images and data analysis done by Maharashtra Remote Sensing Application Centre (MRSAC) and the National Remote Sensing Centre (NRSC) to assess the area of land, and the conditions of select crops in select talukas. However, the second phase includes an analysis of the data collected to build a seamless framework for agriculture modeling and a geospatial database of soil nutrients, rainfall and moisture stress to facilitate location- specific advisories to farmers.

\section{References}

Baruah A. 2019. Artificial intelligence in Indian agriculture- an industry and startup overview. Business Intelligence and Analytics.

Das, S., 2019. Top AI- powered projects in Indian agriculture sector. Analytics India Magazine.

Deer, M., 2020. Artificial intelligence in farming and agriculture. Towards data science.

Department of Science and Technology (DST), gov of India. 2019. New soil monitoring technology can help farming decisions. (dst.gov.in).

Dhanabalan, T. and Sathish, A. 2018. Transforming Indian industries through artificial intelligence and robotics in industry 4.0. International Journal of Mechanical Engineering and Technology. 9(10): 835-845.
Dharmaraj, V. and Vijayanand, C., 2018. Artificial intelligence (AI) in agriculture. International Journal of Current Microbiology and Applied Sciences. 7(12):2122-2128.

Jha, K. et al., 2019. A comprehensive review on automation in agriculture using artificial intelligence. KeAi- Chinese Roots, Global Impact. pp.1-12.

Jivabhumi. (jivabhumi.com)

Mohiuddin, S.M., 2015. Agricultural robotics and its scope in India. International Journal of Engineering Research \& Technology. ISNN:2278-0818. vol.4.

Murugesan, R. et al., 2019. Artificial intelligence and agriculture 5.0. International Journal of Recent Technology and Engineering. 8(2): 1870-1877.

Niti Ayog, 2018. National strategy for artificial intelligence.

Press Information Bureau (PIB), gov of India. 2019. Use of artificial intelligence in agriculture. (pib.gov.in).

Shankar, S., 2017. Gobasco gets funds from Matrix Partners. Economic Times.

Shrivastava, S.K., 2018. Artificial intelligence: way forward for India. Journal of Information Systems and Technology Management., ISSN: 18071775. vol. 15.

Tripathi K., 2019. AI- powered agriculture: govt uses artificial intelligence to boost farming. Financial Express.

\section{How to cite this article:}

Diksha Manaware. 2020. Artificial Intelligence: A New Way to Improve Indian Agriculture. Int.J.Curr.Microbiol.App.Sci. 9(03): 1095-1102. doi: https://doi.org/10.20546/ijcmas.2020.903.128 